\title{
Merging of unequal mass binary black holes in non-axisymmetric galactic nuclei
}

\author{
Peter Berczik $^{1,2,3}$, Long Wang ${ }^{4,1}$, Keigo Nitadori ${ }^{5}$ \\ and Rainer Spurzem ${ }^{1,4,3}$ \\ ${ }^{1}$ National Astronomical Observatories of China, Chinese Academy of Sciences, 20A Datun \\ Rd., Chaoyang District, Beijing 100012, China \\ email: berczik@nao.cas.cn \\ ${ }^{2}$ Main Astronomical Observatory, National Academy of Sciences of Ukraine, 27 Akademika \\ Zabolotnoho St., 03680, Kyiv, Ukraine \\ ${ }^{3}$ Astronomisches Rechen-Institut, Zentrum für Astronomie, University of Heidelberg, \\ Mönchhofstrasse 12-14, 69120, Heidelberg, Germany \\ ${ }^{4}$ Kavli Institute for Astronomy and Astrophysics, Peking University, Beijing 100871, China \\ ${ }^{5}$ RIKEN Advanced Sciennce Institute, Wako, Japan
}

\begin{abstract}
In this work we study the stellar-dynamical hardening of unequal mass massive black hole $(\mathrm{MBH})$ binaries in the central regions of galactic nuclei. We present a comprehensive set of direct $N$-body simulations of the problem, varying both the total mass and the mass ratio of the MBH binary. Our initial model starts as an axisymmetric, rotating galactic nucleus, to describe the situation right after the galaxies have merged, but the black holes are still unbound to each other. We confirm that results presented in earlier works (Berczik et al. 2006; Khan et al. 2013; Wang et al. 2014) about the solution of the "last parsec problem" (sufficiently fast black hole coalescence for black hole growth in cosmological context) are robust for both for the case of unequal black hole masses and large particle numbers. The MBH binary hardening rate depends on the reduced mass ratio through a single parameter function, which quantitatively quite well agrees with standard 3 body scattering theory (see e.g., Hills 1983). Based on our results we conclude that $\mathrm{MBH}$ binaries at high redshifts are expected to merge with a factor of $\sim 2$ more efficiently, which is important to determine the possible overall gravitational wave signals. However, we have not yet fully covered all the possible parameter space, in particular with respect to the preceding of the galaxy mergers, which may lead to a wider variety of initial models, such as initially more oblate and / or even significantly triaxial galactic nuclei. Our $N$-body simulations were carried out on a new special supercomputers using the hardware acceleration with graphic processing units (GPUs).
\end{abstract}

Keywords. black holes, binary black holes, galactic nuclei, stellar dynamics

\section{Introduction}

Supermassive black holes (MBHs) are commonly observed at the centers of most galaxies, and their mass correlates to parameters of the host galaxy, such as its mass or velocity dispersion (see e.g., Gültekin et al. 2009). So, the presence of MBH binaries in galactic centers is an inevitable evolutionary phase during the merger history of galaxies, according to the popular hierarchical structure formation scenario in the $\Lambda$ Cold Dark Matter $(\Lambda \mathrm{CDM})$ paradigm. Binary black holes in galactic nuclei have been searched for by looking for special patterns in radio lobes or jets or quasi-periodic electromagnetic emission in light curves (Komossa 2006).

Originally it was expected that binary black holes would stall at some critical separation (called the "final parsec problem"), which is not small enough for gravitational 
Table 1. Full set of our model runs.

\begin{tabular}{cccccccc}
\hline$\frac{m_{2}}{m_{1}} \times 10^{-2}$ & $\mu \times 10^{-2}$ & $025 \mathrm{k}$ & $050 \mathrm{k}$ & $100 \mathrm{k}$ & $250 \mathrm{k}$ & $400 \mathrm{k}$ & $1 \mathrm{M}$ \\
\hline $0.01 / 1$ & 0.0099 & - & - & 450 & - & - & - \\
$0.02 / 1$ & 0.0196 & - & - & 450 & - & - & - \\
$0.03 / 1$ & 0.0291 & - & - & 350 & - & - & - \\
$0.05 / 1$ & 0.0476 & - & - & 350 & - & - & - \\
$0.10 / 1$ & 0.0909 & 250 & 250 & $250^{*}$ & 250 & 250 & 150 \\
$0.20 / 1$ & 0.1666 & 250 & 250 & $250^{*}$ & 250 & 250 & 150 \\
$0.50 / 1$ & 0.3333 & 250 & 250 & $250^{*}$ & 250 & 250 & 150 \\
$1.00 / 1$ & 0.5000 & 250 & 250 & $250^{*}$ & 250 & 250 & 150 \\
\hline $0.02 / 2$ & 0.0198 & - & - & 450 & - & - & - \\
$0.03 / 2$ & 0.0295 & - & - & 450 & - & - & - \\
$0.05 / 2$ & 0.0488 & - & - & 350 & - & - & - \\
$0.10 / 2$ & 0.0952 & - & - & 350 & - & - & - \\
$0.20 / 2$ & 0.1818 & - & - & 250 & - & - & - \\
$0.40 / 2$ & 0.3333 & - & - & 250 & - & - & - \\
$1.00 / 2$ & 0.6666 & 250 & 250 & 250 & 250 & 250 & 150 \\
$2.00 / 2$ & 1.0000 & 250 & 250 & 250 & 250 & 250 & 150 \\
\hline $0.04 / 4$ & 0.0396 & - & - & 450 & - & - & - \\
$0.08 / 4$ & 0.0784 & - & - & 450 & - & - & - \\
$0.10 / 4$ & 0.0976 & - & - & 350 & - & - & - \\
$0.20 / 4$ & 0.1905 & - & - & 350 & - & - & - \\
$0.40 / 4$ & 0.3636 & - & - & 250 & - & - & - \\
$0.80 / 4$ & 0.6666 & - & - & 250 & - & - & - \\
$2.00 / 4$ & 1.3333 & 250 & 250 & 250 & 250 & 250 & 150 \\
$4.00 / 4$ & 2.0000 & 250 & 250 & 250 & 250 & 250 & 150 \\
\hline
\end{tabular}

Notes: First column: MBH masses in model units. Columns 3 - 8: Particle number in the stellar galactic nucleus. The first column gives the $\mathrm{MBH}$ masses and the second column the $\mathrm{MBH}$ reduced mass $\mu$.

radiation induced orbit shrinking and coalescence to set in Begelman et al. (1980); Makino et al. (1993); Makino (1997); Hemsendorf et al. (2002); Berczik et al. (2005); Merritt (2006). However, there is a growing evidence that in the reality of galaxy mergers, where galaxies are axisymmetric or even triaxial, there is no problem to refill the loss-cone and the binary black hole hardening rate becomes independent of the particle number used in the simulation (Yu 2002; Merritt \& Poon 2004; Berczik et al. 2006). This process will overcome the final parsec problem (Preto et al. 2009; Khan et al. 2011, 2013) and it seems that the previously studied spherically symmetric galactic nuclei actually were some worst case scenario (Preto et al. 2011). Berentzen et al. (2009) have demonstrated that the new more realistic models make it possible to bring an $\mathrm{MBH}$ binary to final relativistic coalescence in a short time scale.

\section{Numerical model and initial conditions}

The simulations presented in this work have been carried out using the direct $N$-body code $\varphi$-GPU (Berczik et al. 2011, 2013). The basic concepts of the code are based on the publicly available $\varphi$-GRAPE (Harfst et al. 2007) code, an Aarseth $N$-body 1-like code including an efficient MPI parallelization and support for the special-purpose hardware GRAPE (Fukushige et al. 1991) (currently Graphic Processing Units - GPU). The current version of the $\varphi$-GPU code has been fully rewritten in the $\mathrm{C}++$ programming language for the most effective use of GPUs. It supports the use Hermite integration algorithms of $4^{t h}, 6^{t h}$ or $8^{t h}$ ) order. Furthermore, it includes a hierarchical block 

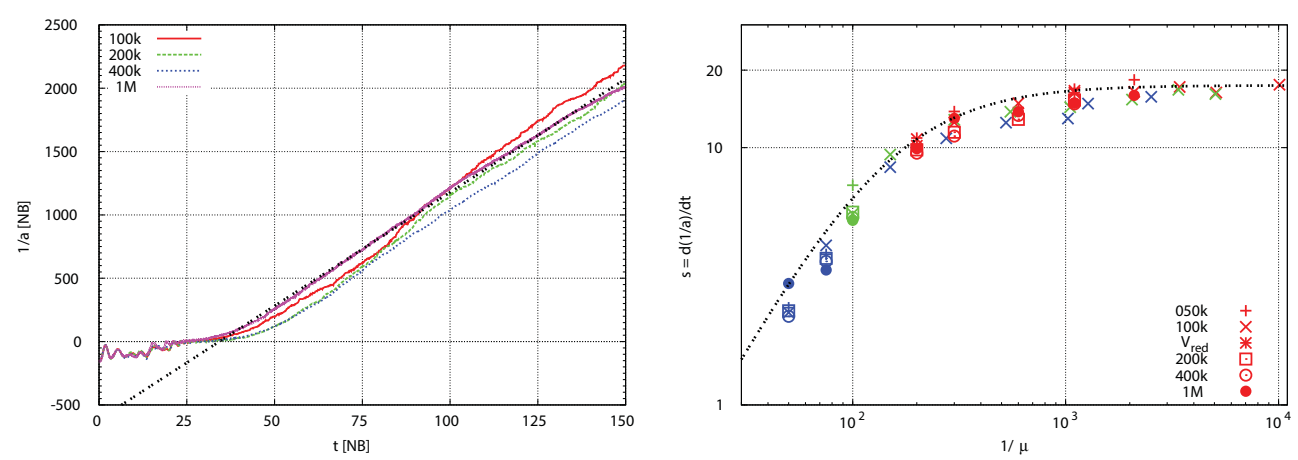

Figure 1. Evolution of the MBH binary hardening rate. The left panel shows the method of hardening determination based on the particular set of the runs. The right panel shows the hardening rate dependency from the reduced mass of the MBH binary system.

time-step scheme. Compared to the earlier $\varphi$-GRAPE (Harfst et al. 2007) implementation we obtain an additional speed-up a factor of $\times 2$ on the recent generation of NVIDIA GPUs (i.e., M2050 and K20 cards), depending on the specific number of particles and computing nodes used. Further details on this high-performance computing code will be described in papers (Berczik et al. 2011, 2013). The present code is well tested and already used to obtain important results in our earlier large scale few million body simulation (Khan et al. 2012).

We scale the numerical units of our initial models applying the standard $N$-body normalization (Aarseth et al. 1974) by setting both the gravitational constant $G$ and the total mass of the stellar nucleus system to unity. The total energy of the system is set to $E=-\frac{1}{4}$.

The initial conditions of our $N$-body simulations presented here are based on the ones used in Berczik et al. (2006) and Berentzen et al. (2009). The initial stellar galactic nucleus follows a distribution function for a rotating King model (see, e.g., Einsel \& Spurzem 1999, and references therein). The concentration and rotation parameter are set to $W_{0}=6$ and to $\omega_{0}=1.8$, respectively, in all the models. The total angular momentum vector of both the stellar nucleus and the MBHs are aligned with the $z$-axis of our coordinate frame. We place the two MBHs in the $z=0$ mid-plane with initial coordinate components $x_{1,2}=0$ and $y_{1,2}= \pm 0.3$, where the sub-scripts denotes the two black hole particles.

The MBH masses and particle numbers with which the stellar system has been realized are given in Table 1 . The initial $x$-velocity of the MBHs in these simulations has been chosen to be $v_{x ; 1,2}= \pm V_{\text {circ }}$, where $V_{\text {circ }}$ is the circular velocity within the stellar background model. With our choice of initial parameter the circular velocity, in $N$-body units, is $V_{\text {circ }}=0.7$ at the initial distance. In Table 1., we also show with mark $(*)$ the extra simulations which have been run, but this time with and initial MBH velocity of $v_{x ; 1,2}= \pm 0.1 \times V_{\text {circ }}=0.07$. We choose this value to have a faster pairing of the two $\mathrm{MBH}$ due to a more eccentric initial orbit.

\section{Results and discussion}

An example of the typical binary evolution in our simulations is shown in Fig. 1 (left panel) in terms of the inverse binary's semi-major $a$ as a function of time. From the slope of a linear fit to $1 / a(t)$ we then determine the hardening rate $\mathrm{d}(1 / a) / \mathrm{d} t$ of the binary. 
In Fig. 1 (right panel) we show the hardening rate as a function of reduced mass of the $\mathrm{MBH}$ binary $\mu$. As we can see from the figure the hardening rate for our set of models and parameters are only a single function of the $\mu$ (or actually the $1 / \mu$ ).

Our results have strong implications on the merger rate and history of MBHs and on the $\mathrm{MBH}$ growth in the cosmological context. Numerical $\Lambda$ CDM simulations show that the galaxy merger rate in the early universe is higher than at low redshifts $z$. If at high redshift we have a higher galaxy merger rate and also the less massive MBH binaries (i.e., total binary mass and also a reduced mass are both smaller) we can get a significantly higher (few times) hardening rate at early universe. These rates (as we already see in Berczik et al. (2006); Berentzen et al. (2008)) are high enough to reach the relativistic regime and merge efficiently.

PB thanks the International Astronomical Union for the grant support which give him the opportunity to participate on the IAU Symposium No. 312 in Beijing-2014.

\section{References}

Aarseth, S. J., Henon, M., \& Wielen, R. 1974, Astron. and Astroph., 37, 183

Begelman, M. C., Blandford, R. D., \& Rees, M. J. 1980, Nature, 287, 307

Berczik, P., Merritt, D., \& Spurzem, R. 2005, ApJ, 633, 680

Berczik, P., Merritt, D., Spurzem, R., \& Bischof, H.-P. 2006, ApJL, 642, L21

Berczik, P., Spurzem, R., Wang, L., Zhong, S., \& Huang, S. 2013, in Third International Conference "High Performance Computing", HPC-UA 2013, p. 52-59, 52-59

Berczik, P., Nitadori, K., Zhong, S., et al. 2011, in International conference on High Performance Computing, Kyiv, Ukraine, October 8-10, 2011., p. 8-18, 8-18

Berentzen, I., Preto, M., Berczik, P., Merritt, D., \& Spurzem, R. 2008, AN, 329, 904

-. 2009, ApJ, 695, 455

Einsel, C. \& Spurzem, R. 1999, MNRAS, 302, 81

Fukushige, T., Ito, T., Makino, J., et al. 1991, Publ. Aston. Soc. Jpn., 43, 841

Gültekin, K., Richstone, D. O., Gebhardt, K., et al. 2009, ApJ, 698, 198

Harfst, S., Gualandris, A., Merritt, D., et al. 2007, N. Astr., 12, 357

Hemsendorf, M., Sigurdsson, S., \& Spurzem, R. 2002, ApJ, 581, 1256

Hills, J. G. 1983, Astron. J., 88, 1269

Khan, F. M., Berentzen, I., Berczik, P., et al. 2012, ApJ, 756, 30

Khan, F. M., Holley-Bockelmann, K., Berczik, P., \& Just, A. 2013, ApJ, 773, 100

Khan, F. M., Just, A., \& Merritt, D. 2011, ApJ, 732, 89

Komossa, S. 2006, Mem. Soc. Astron. Italiana, 77, 733

Makino, J. 1997, ApJ, 478, 58

Makino, J., Fukushige, T., Okumura, S. K., \& Ebisuzaki, T. 1993, Publ. Aston. Soc. Jpn., 45, 303

Merritt, D. 2006, Reports on Progress in Physics, 69, 2513

Merritt, D. \& Poon, M. Y. 2004, ApJ, 606, 788

Preto, M., Berentzen, I., Berczik, P., Merritt, D., \& Spurzem, R. 2009, Journal of Physics Conference Series, 154, 012049

Preto, M., Berentzen, I., Berczik, P., \& Spurzem, R. 2011, ApJL, 732, L26

Wang, L., Berczik, P., Spurzem, R., \& Kouwenhoven, M. B. N. 2014, ApJ, 780, 164

$\mathrm{Yu}, \mathrm{Q} .2002, M N R A S, 331,935$ 